SPIE Optical Metrology 2011

\title{
Remote Laboratory for Digital Holographic Metrology
}

Marc Wilke, Guohai Situ, Igor Aleksenko, Margarita Riedel, Giancarlo Pedrini, Sabina Jeschke and Wolgang Osten

\author{
Institut für Technische Optik \\ Universität Stuttgart, Germany
}




\section{Motivation}

- Scientists wish to collaborate on research

- Raw data is already often recorded and analyzed using computers

- Conditions of the measurement process need to be recorded to interpret the raw data (traditionally in a lab book)

- Publication of raw data desirable 


\section{Motivation}

cito

\section{Science and Research}

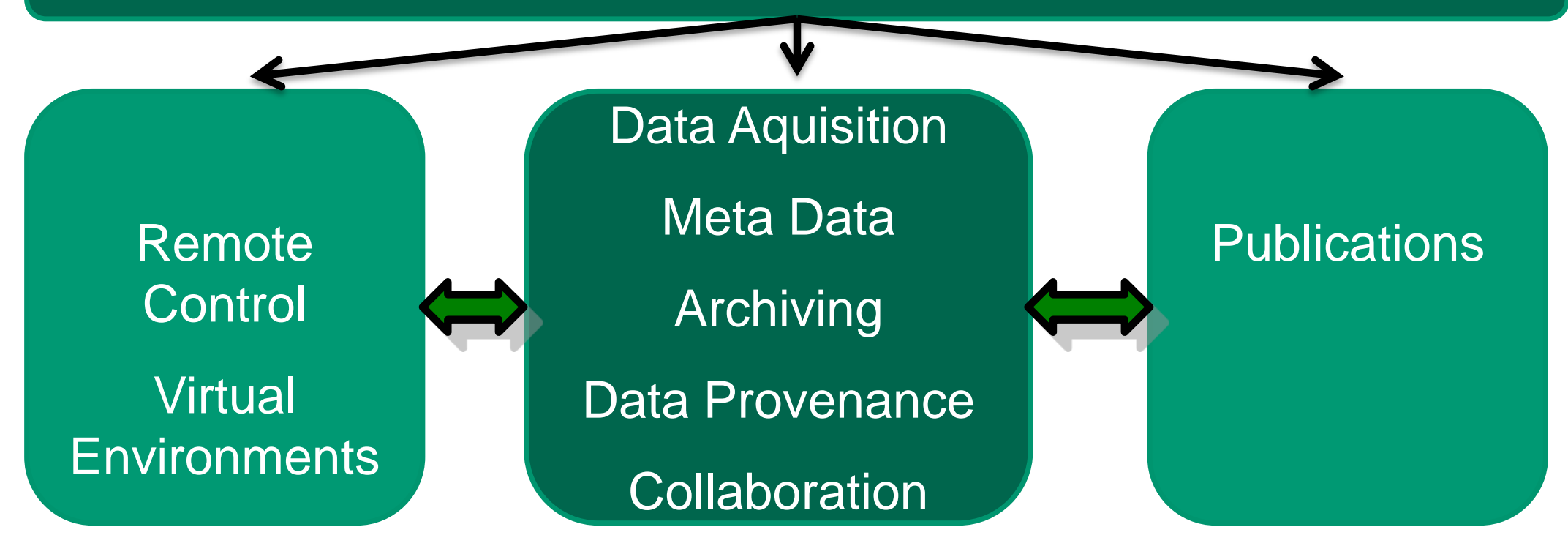




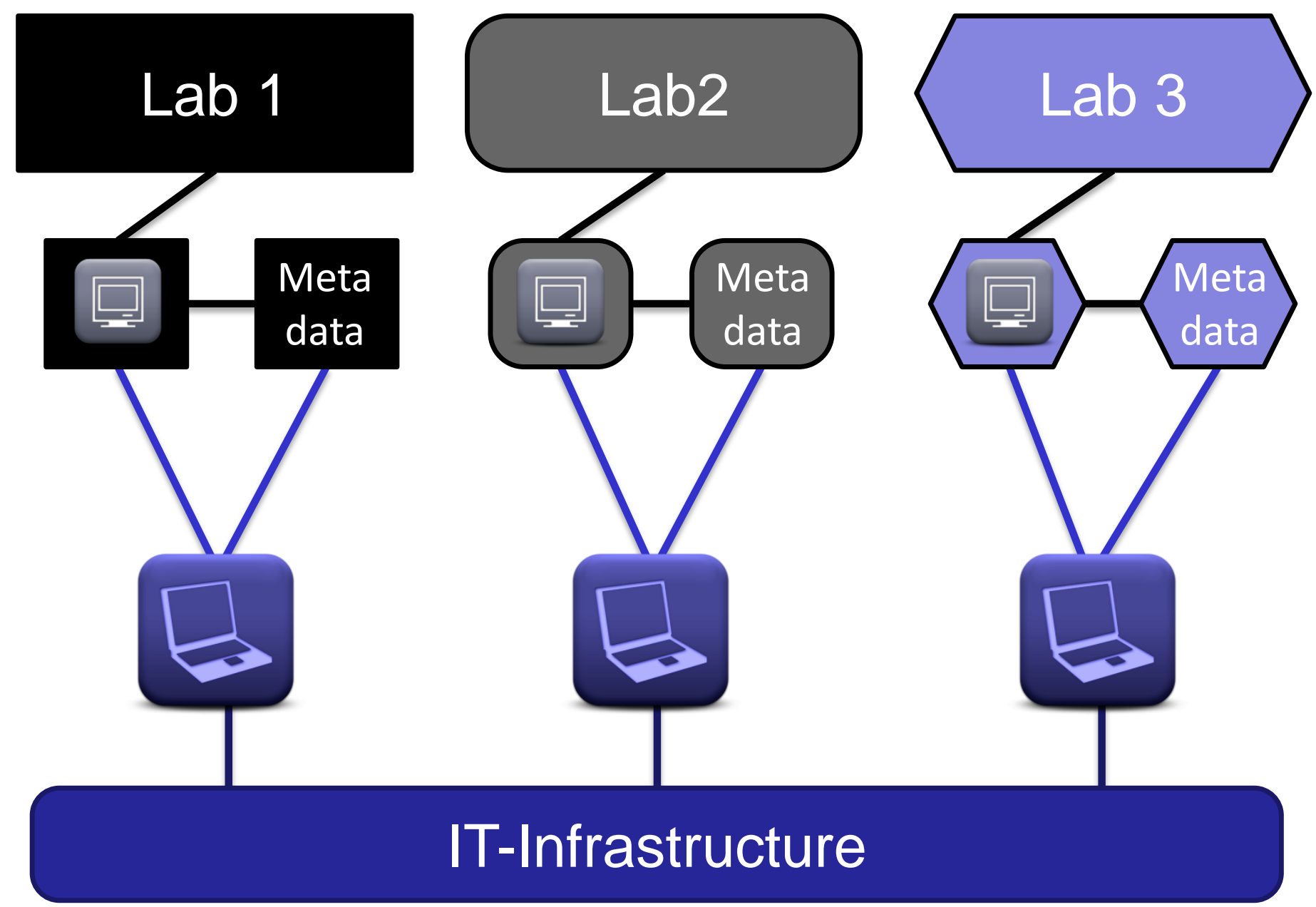




\section{Aim of BW-eLabs}

- Design an Infrastructure for automatic recording, storage and access to research data

- Secure, single sign-on access to labs without loss of local control

- Support of publication process, including publication of raw data

- Support for integration of specific labs 


\section{BW-eLabs Architecture}

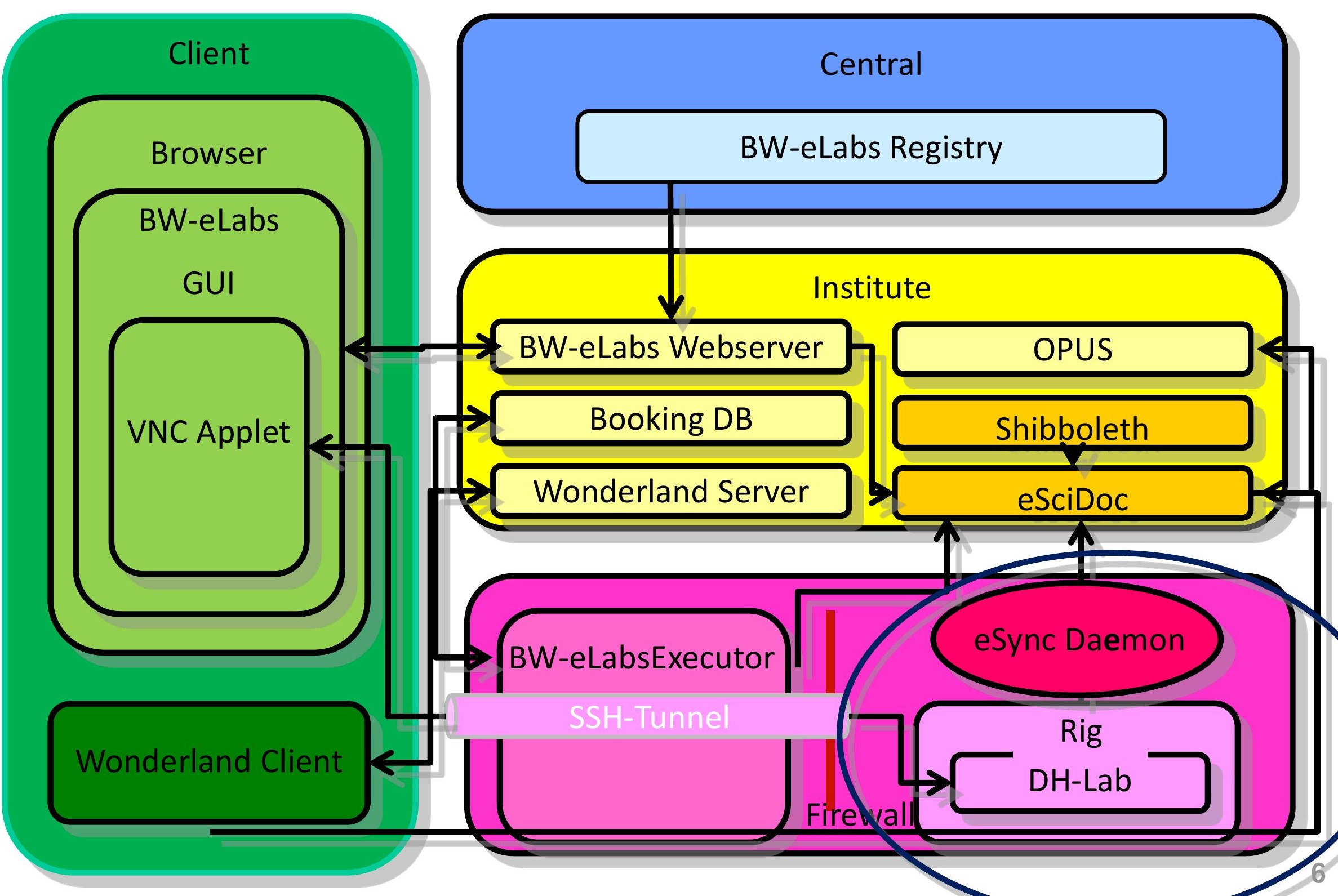




\section{Dataflow in BW-eLabs}

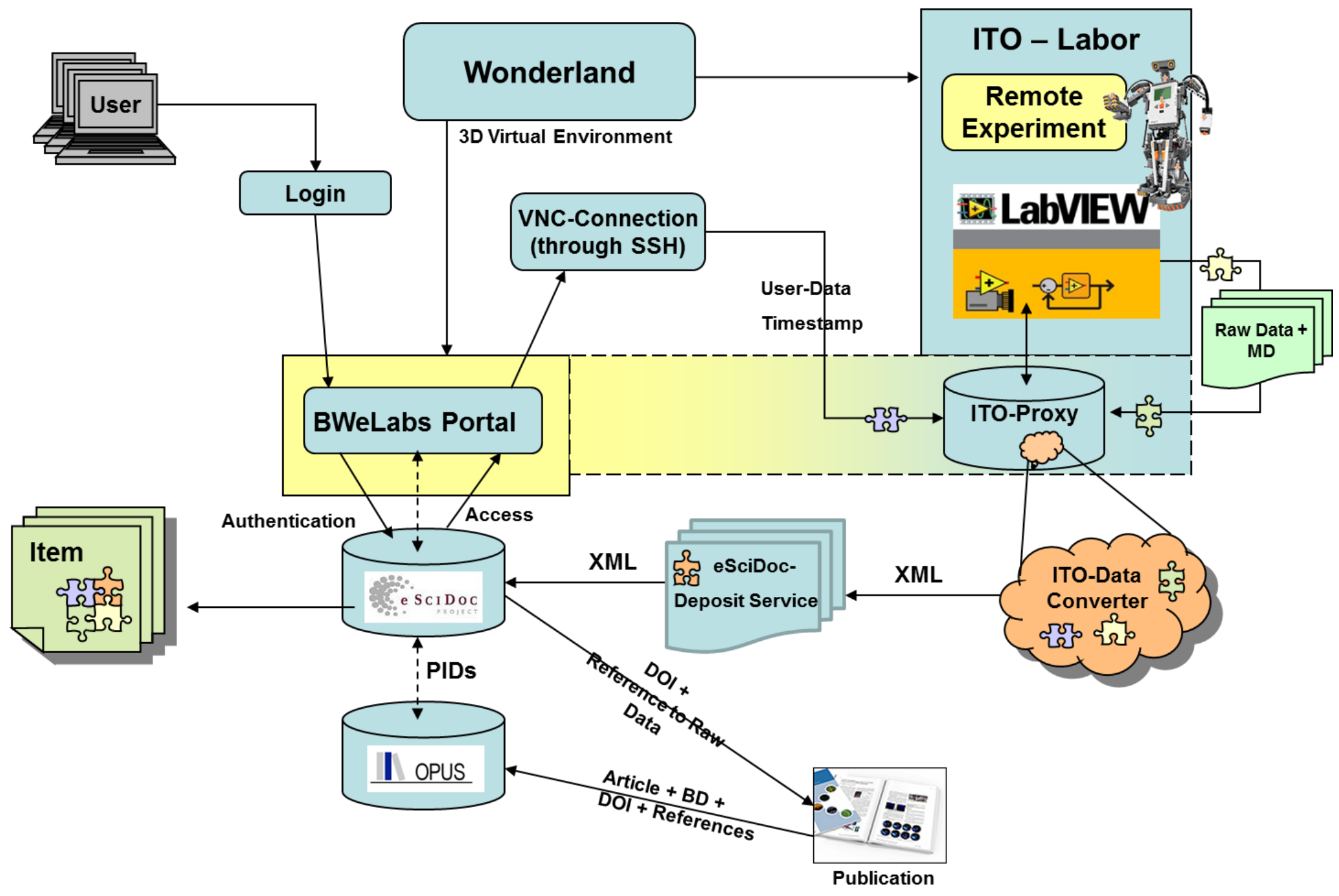




\section{DH Lab - Setup}

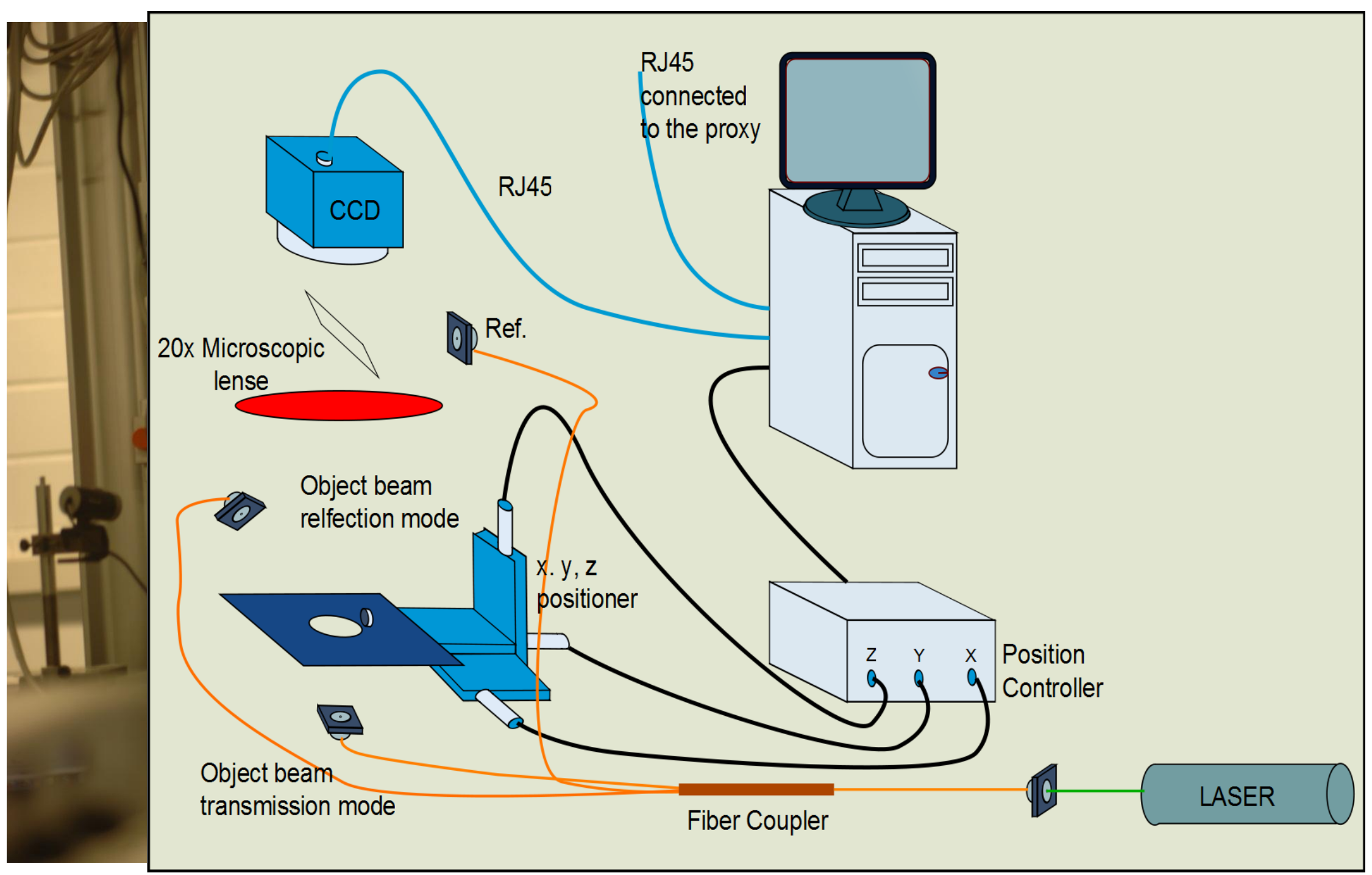




\section{DH Lab}

- Hologram stores all optical information of an object Well suited for remote collaboration

- Complete state of the system can be saved and restored automatically (including geometry and position, reconstruction, description of experiment, description of Holograms)

- Searchable Meta Data generated and stored automatically in eSciDoc database 


\section{Results and Future Work}

- BW-eLabs implements an infrastructure for handling research data

- DH Lab implemented as a demonstrator

- Low level integration of additional labs already feasible through generic interfaces (VNC, eSync Daemon, eSciDoc Deposit Service)

- Future Work: 3D Interface in Wonderland, publication of raw data 


\section{Acknowledgement}

- Funded by the Ministerium für Wissenschaft, Forschung und Kunst, Baden-Württemberg under the „BW-eLabs“ Project

- Projectpartners:

- Universität Stuttgart (RUS, IITS, ITO, Bibliothek)

- FIZ Karlsruhe (Fachinformationszentrum)

- Freiburger Materialforschungszentrum (FMF) und $\mathrm{RZ}$ der Universität Freiburg

- Hochschule der Medien 


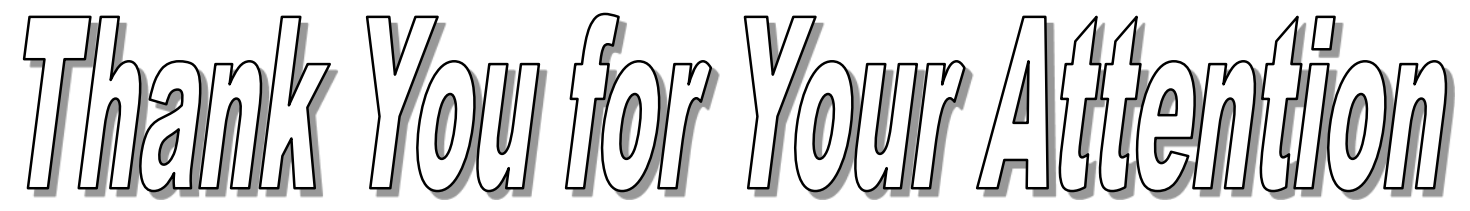

